\title{
OUTER CONJUGACY CLASSES OF TRACE SCALING AUTOMORPHISMS OF STABLE UHF ALGEBRAS
}

\author{
GEORGE A. ELLIOTT, DAVID E. EVANS and AKITAKA KISHIMOTO
}

\section{Introduction.}

Connes [C] classified automorphisms of the $\mathrm{II}_{\infty}$ approximately finite-dimensional (AFD) factor $R_{0,1}$ up to outer conjugacy. An automorphism $\theta$ of $R_{0,1}$ determines a modulus or scaling factor $\lambda$ on a trace $\tau, \tau \theta=\lambda \tau$. It follows from this classification and [CT] that automorphisms which do not leave a trace invariant are classified up to conjugacy by their modulus; for each $\lambda \in(0,1)$ there is up to conjugacy a unique automorphism $\theta_{\lambda}$ of $R_{0,1}$ which scales the trace by $\lambda$. Consequently, for such $\lambda$, there is a unique factor of type $\mathrm{III}_{\lambda}$, the Powers factor $R_{\lambda}$, whose associated type $\mathrm{II}_{\infty}$ factor is $R_{0,1}$; necessarily, $R_{\lambda}=R_{0,1} \rtimes_{\theta_{\lambda}} \mathrm{Z}$.

A key idea for model building in Connes's work on the classification of automorphisms of AFD factors [C] and in subsequent work of Jones [J], Ocneanu [O], and Kawahigashi, Sutherland and Takesaki [KST] on amenable group actions is a non-commutative Rohlin lemma for an aperiodic automorphism of a finite von Neumann algebra. Recall that an automorphism $\theta$ of a von Neumann algebra $M$ is said to be properly outer if the restriction to $M_{e}$ is outer for each non-zero invariant projection $e$, and aperiodic if every non-zero power is properly outer. Connes showed that an automorphism $\theta$ of $M$ is properly outer if and only if for each non-zero projection $e$ in $M$, and $\varepsilon>0$, there exists a non-zero projection $f$ in $M$ such that $\|f \theta(f)\|<\varepsilon$ and $f \leq e$. With an abundance of such projections, whose translates are almost orthogonal, Connes could deduce a non-commutative Rohlin lemma. If $\theta$ is an aperiodic automorphism of a finite (not necessarily separable) von Neumann algebra leaving invariant a normalised faithful normal trace $\tau$, then for each positive integer $n$ and each $\varepsilon>0$, there exist projections $F_{1}, \cdots, F_{n}$ in $M$ such that $\sum_{j=1}^{n} F_{j}=1$ and $\left\|\theta\left(F_{j}\right)-F_{j+1}\right\|_{2} \leq \varepsilon$, $j=1, \ldots, n$, where $F_{n+1}=F_{1}$ and $\|x\|_{2}=\tau\left(x^{*} x\right)^{\frac{1}{2}}$.

Received February 2, 1996; in revised form February 28, 1996. 
The Rohlin lemma can be applied to study an automorphism $\theta$ of a von Neumann algebra $M$ which is not necessarily finite through the algebra of central sequences which may be of type $\mathrm{II}_{1}$. If $\omega$ is an ultrafilter on $\mathrm{N}$, let $M_{\omega}$ denote the asymptotic centraliser (see [C]), the von Neumann algebra of centralising sequences (bounded sequences $\left(x_{n}\right)$ such that $\lim _{n \rightarrow \omega}\left\|\left[x_{n}, \psi\right]\right\|=0$ for all $\psi \in M_{*}$ ), modulo $\omega$-null sequences (bounded sequences converging*strongly to zero). Then $M \cong M \otimes R_{0}$ if and only if $M_{\omega}$ is of type $\mathrm{II}_{1}$ (in this case $M$ is said to be strongly stable), where $R_{0}$ is the hyperfinite $\mathrm{II}_{1}$ factor, and the Rohlin lemma may be applied to the induced automorphism $\theta_{\omega}$ of $M_{\omega}$. From the Rohlin lemma, one can deduce a stability or 1-cohomology property. In the algebra of sequences, this takes the form that if $\theta$ is an automorphism of a strongly stable factor and $\theta_{\omega}^{n} \neq 1$ for all $n \neq 0$, then $\theta_{\omega}$ is stable in the sense that any unitary of $M_{\omega}$ is of the form $v \theta_{\omega}\left(v^{*}\right)$ for some unitary $v$ in $M_{\omega}$.

These ideas concerning the Rohlin lemma and stability were taken over to a $C^{*}$-algebraic setting in [HJ1], [HJ2], and [HO] to classify product cyclic actions on UHF $C^{*}$-algebras $A$ up to outer conjugacy and conjugacy. In this case the appropriate sequence algebra $A^{\infty}$ is $l^{\infty}(\mathrm{N}, A)$ modulo sequences which converge to zero in norm, and the algebra of central sequences is $A_{\infty}=A^{\infty} \cap A^{\prime}$. These ideas concerning the Rohlin property and stability were taken further in [R1], [R2], in the programme to classify separable amenable $C^{*}$-algebras by $K$-theoretic invariants.

The Rohlin property on which these and later articles were based was that established in [BKRS] for the shift on the Pauli algebra $M_{2 \infty}=\bigotimes_{z} M_{2}$. This was obtained via quasi-free techniques, applied to the quasi-free shift on the (even) Fermion algebra. The Rohlin property for the shift on $M_{2^{\infty}} \mathrm{im}-$ mediately implies the Rohlin property for the shift on the UHF algebra $M_{(2 n)^{\infty}}=M_{2^{\infty}} \otimes M_{n^{\infty}}$. A weak Rohlin property was established in [BEK1] for the shift on an odd UHF algebra $M_{m \infty}$ (where $m$ is odd) via the embedding [CE] of the gauge-invariant Fermion algebra in the UHF algebra $M_{m^{\infty}}$, which is compatible with the shift. In [K1], it was shown that this weaker form of the Rohlin property actually guaranteed the Rohlin property itself for the shift on $M_{m^{\infty}}$ for an arbitrary $m$.

Furthermore, it was shown in [K2] that an automorphism of an arbitrary UHF algebra has the Rohlin property if and only if every non-zero power is uniformly outer (i.e., the weak extension is outer in the tracial representation), or, equivalently, the crossed product $A \rtimes \mathrm{Z}$ has a unique tracial state, or the crossed product is of real rank zero. It was also shown that all such automorphisms are outer conjugate (e.g. to an infinite tensor product automorphism $\left(\bigotimes_{n=1}^{\infty} M_{m_{n}}, \bigotimes_{n=1}^{\infty} \operatorname{Ad} U_{n}\right)$ with the eigenvalues of $\bigotimes_{n=l}^{\infty} U_{n}$ being uniformly distributed for any $l$ ). Hence the crossed products are all iso- 
morphic and $A \mathrm{~T}$ - inductive limits of direct sums of matrix algebras over the continuous functions on the circle.

Here we shall show that trace scaling automorphisms of a stable UHF algebra, with the same non-trivial scaling factor, are outer conjugate. An automorphism of a stable UHF algebra $B \otimes \mathcal{K}$, with $B$ a unital UHF algebra, which scales the trace by $p / q \neq 1$ with $p$ and $q$ relatively prime, induces a partial endomorphism $\gamma$, an isomorphism of $B \cap C_{1}^{\prime}$ onto $B \cap C_{2}^{\prime}$ where $C_{1}$ and $C_{2}$ are subalgebras of $B$ isomorphic to $M_{p}$ and $M_{q}$. This partial endomorphism $\gamma$ extends to a partial endomorphism of $R$, the hyperfinite factor generated by $B$ in its tracial representation, taking $R \cap C_{1}^{\prime}$ to $R \cap C_{2}^{\prime}$. This determines an automorphism $\gamma_{\omega}$ of $R_{\omega}$. Lemma 1 shows that $\gamma$ is outer in the sense that for each full matrix algebra $N$ of $R$ containing $C_{1}$, every non-zero projection $f$ in $R \cap N^{\prime}$, and any unitary $U$ in $R$ the following holds:

$$
\inf \left\{\|e U \gamma(e)\| ; 0 \neq e \leq f, e=e^{2}=e^{*} \in R \cap N^{\prime}\right\}=0 .
$$

It then follows (Lemma 2) that any non-zero power of the automorphism $\gamma_{\omega}$ is properly outer, that the partial endomorphism $\gamma$ of the von Neumann algebra $R$ has the Rohlin property (Lemma 3), and, hence, that the partial endomorphism $\gamma$ on the $C^{*}$-algebra $B$ has the Rohlin property (Lemma 4 ). From this, stability of the partial endomorphism $\gamma$ on $B$ can be deduced (Lemma 5), from which $\gamma$ is seen to be outer conjugate with $\gamma \otimes \sigma$ for any automorphism $\sigma$ of the UHF algebra $M_{(p q)^{\infty}}=B_{0}$ (note $B \cong B \otimes B_{0}$ ). Arguments parallel to those in the von Neumann algebra setting then yield that all trace scaling automorphisms of the stable UHF algebra $A$, with the same non-trivial scaling factor $p / q \neq 1$, are outer conjugate. The classification of simple purely infinite amenable $C^{*}$-algebras which satisfy the UCT ([R2], [Kir], [Phi]) shows that if $A=M_{(p q)} \otimes \mathcal{K}$ the crossed product $A \rtimes \mathrm{Z}$ is isomorphic to the stable Cuntz algebra $O_{|p-q|+1} \otimes \mathcal{K}$, if $p$ and $q$ are relatively prime.

The arguments here in fact establish the stronger assertion (Corollary 8) that unital endomorphisms of a UHF algebra with image the commutant of a non-trivial matrix algebra are classified up to outer conjugacy by the order of that algebra, and similarly for unital endomorphisms of the hyperfinite factor of type $\mathrm{II}_{1}$.

This work was initiated at the Fields Institute for Research in Mathematical Sciences, in July and August of 1995. AK is grateful to M. Takesaki for explaining the solution of the present problem in the factor case. DEE was supported by the NSERC - Royal Society Anglo-Canadian Exchange Scheme and AK by the NSERC - JSPS Canadian-Japanese Exchange Scheme. The work was completed in September and October of 1995 when AK was visiting the University of Wales, Swansea, supported by JSPS and 
the Royal Society under the Bilateral Programme between the Isaac Newton Research Institute for Mathematical Sciences, Cambridge, and the Research Institute for Mathematical Sciences, Kyoto University.

\section{The Main Results.}

Let $A$ be a stable UHF algebra; that is, let $A$ be a $C^{*}$-algebra isomorphic to $B \otimes \mathcal{K}$ where $B$ is a unital UHF algebra and $\mathcal{K}$ is the algebra of compact operators on an infinite-dimensional separable Hilbert space. There is a densely defined lower semi-continuous trace $\tau$ on $A ; \tau$ is unique up to constant multiple. Let $\alpha$ be an automorphism of $A$. We define $s(\alpha) \in \mathrm{R}$ by $\tau \alpha=s(\alpha) \tau$. It follows that $s(\alpha)$ is a positive rational number and the map $\alpha_{*}$ on $K_{0}(A)$ is multiplication by $s(\alpha)$, identifying $K_{0}(A)$ with a dense subgroup of $\mathrm{R}$ containing $\mathrm{Z}$. We shall say that automorphisms $\alpha$ and $\beta$ of $A$ are outer conjugate if there is an automorphism $\sigma$ of $A$ and a unitary $U$ in the multiplier algebra $M(A)$ of $A$ such that $\alpha=(\operatorname{Ad} U) \sigma \beta \sigma^{-1}$. If $\alpha$ and $\beta$ are outer conjugate then $s(\alpha)=s(\beta)$.

Let $\alpha$ be an automorphism of $A$ such that $s(\alpha) \neq 1$. Let $s(\alpha)=p / q$ where $p$ and $q$ are relatively prime positive integers. Since $K_{0}(A)=(p / q) K_{0}(A)$, it follows that

$$
K_{0}(A)=K_{0}(A) \otimes \mathbf{Z}[1 / p q] .
$$

That is, $A$ is isomorphic to $A \otimes M_{(p q)^{\infty}}$ where $M_{n^{\infty}}$ is the (unital) UHF algebra of type $n^{\infty}$.

Let $e$ be a projection in $A$ whose equivalence class is 1 in $K_{0}(A)$, and set $B=e A e$. Then $A \cong B \otimes \mathcal{K}$ and $B \cong M_{(p q)^{\infty}} \otimes D$, where $D$ is a UHF algebra (or a matrix algebra) such that $K_{0}(D)$ is not divisible by $p$ or $q$. Let us identify $B$ with $M_{(p q)^{\infty}} \otimes D$ and choose a subalgebra $C_{1}$ of $M_{(p q)^{\infty}} \otimes 1$ isomorphic to $M_{p}$ and a subalgebra $C_{2}$ of $M_{(p q)^{\infty}} \otimes 1$ isomorphic to $M_{q}$. Set $B_{i}=B \cap C_{i}^{\prime}$. Then $B_{1}$ and $B_{2}$ are isomorphic to $B$. Let us identify $B$ with $B_{1} \otimes C_{1}$ and with $B_{2} \otimes C_{2}$.

Since the minimal projections in $C_{1} \otimes \mathcal{K}(\subset B \otimes \mathcal{K})$ correspond to $1 / p$ in $K_{0}(A)$, the minimal projections in $\alpha\left(C_{1} \otimes \mathcal{K}\right)(\subset B \otimes \mathcal{K})$ correspond to $1 / q$. Hence there is a unitary $U$ in $M(B \otimes \mathcal{K})$ such that

$$
(\operatorname{Ad} U) \alpha\left(C_{1} \otimes \mathcal{K}\right)=C_{2} \otimes \mathcal{K} .
$$

Let us denote by $\bar{\alpha}$ the extension of $\alpha$ to an automorphism of $M(A)$. Then it follows that $(\operatorname{Ad} U) \bar{\alpha}$ maps $B_{1}\left(\cong B_{1} \otimes 1 \subset M(B \otimes \mathcal{K})\right)$ onto $B_{2}$. Set

$$
\begin{aligned}
& \theta=(\operatorname{Ad} U) \alpha \mid C_{1} \otimes \mathcal{K}, \\
& \gamma=(\operatorname{Ad} U) \bar{\alpha} \mid B_{1} .
\end{aligned}
$$


Then $\theta$ is an isomorphism of $C_{1} \otimes \mathcal{K}$ onto $C_{2} \otimes \mathcal{K}$ - note that such an isomorphism is unique up to inner automorphisms (arising from unitaries in $\left.M\left(C_{2} \otimes \mathcal{K}\right)\right)-$ and $\gamma$ is an isomorphism of $B_{1}$ onto $B_{2}$. Note that $(\operatorname{Ad} U) \alpha$ is obtained as follows :

$$
B \otimes \mathcal{K}=B_{1} \otimes\left(C_{1} \otimes \mathcal{K}\right) \stackrel{\gamma \otimes \theta}{\longrightarrow} B_{2} \otimes\left(C_{2} \otimes \mathcal{K}\right)=B \otimes \mathcal{K}
$$

Lemma 1. Let $R$ denote the AFD factor of type $\mathrm{II}_{1}$ and let $C_{1}$ and $C_{2}$ be unital finite-dimensional subalgebras of $R$ such that $C_{1} \cong M_{p}$ and $C_{2} \cong M_{q}$ with $p \neq q$. Let $\gamma$ be an isomorphism of $R \cap C_{1}^{\prime}$ onto $R \cap C_{2}^{\prime}$. Then for any full matrix subalgebra $N$ of $R$ with $N \supset C_{1}$, for any non-zero projection $f$ in $R \cap N^{\prime}$ and for any unitary $U$ in $R$ it follows that

$$
\inf \left\{\|e U \gamma(e)\| ; e \leq f, 0 \neq e=e^{2}=e^{*} \in R \cap N^{\prime}\right\}=0 .
$$

Proof. Let $\theta$ be an isomorphism of $C_{1} \otimes I_{\infty}$ onto $C_{2} \otimes I_{\infty}$ where $I_{\infty}$ is a factor of type $\mathrm{I}_{\infty}$. Then one can define an automorphism $\alpha$ of the von Neumann algebra tensor product $R \otimes I_{\infty}$ by

$$
R \otimes I_{\infty}=R \cap C_{1}^{\prime} \otimes C_{1} \otimes I_{\infty} \stackrel{\gamma \otimes \theta}{\longrightarrow} R \cap C_{2}^{\prime} \otimes C_{2} \otimes I_{\infty}=R \otimes I_{\infty} .
$$

Since $\tau \alpha=(p / q) \tau$ where $\tau$ is a trace on $R \otimes I_{\infty}, \alpha$ is (properly) outer.

Let $N, f$ and $U$ be given. Replacing $\gamma$ by $(\operatorname{Ad} U) \gamma$ and $C_{2}$ by $(\operatorname{Ad} U) C_{2}$ we may suppose that $U=1$. Let $p_{i}$ (resp. $p$ ) be a minimal projection in $C_{i}$ (resp $\left.I_{\infty}\right)$. We may assume that $\theta\left(p_{1} \otimes p\right)=p_{2} \otimes p$. Let $q_{1}$ be a minimal projection in $N \cap C_{1}^{\prime}$ and set $F$ equal to $f p_{1} q_{1} \otimes p$, which is a projection in $R \otimes I_{\infty}$. Let a projection $E \in R \otimes I_{\infty}$ with $E \leq F$ be given. Necessarily, $E$ is of the form $e p_{1} q_{1} \otimes p$ where $e$ is a projection in $R \cap N^{\prime}$. If $N \cong M_{r p}$, then there is a family $\left(V_{1 k} ; k=1, \ldots, r p\right)$ of unitaries in $N$ such that

$$
\sum_{k} V_{1 k} p_{1} q_{1} V_{1 k}^{*}=1
$$

There is also a family $\left(V_{2 k} ; k=1, \ldots, r q\right)$ of unitaries in $\gamma\left(N \cap C_{1}^{\prime}\right) \vee C_{2}$ such that

$$
\sum_{k} V_{2 k} p_{2} q_{2} V_{2 k}^{*}=1
$$

where $q_{2}=\gamma\left(q_{1}\right)$. It follows that

$$
\sum_{k, l} V_{1 k} E V_{1 k}^{*} V_{2 l} \alpha(E) V_{2 l}^{*}=e \gamma(e) \otimes p .
$$


Hence,

$$
\|e \gamma(e)\| \leq p q r^{2} \max \left\{\left\|E V_{1 k}^{*} V_{2 l} \alpha(E)\right\| ; k=1, \ldots, r p, l=1, \ldots, r q\right\} .
$$

By [C, 1.2.2], the infimum of the right hand side over non-zero projections $E \in R \otimes I_{\infty}$ with $E \leq F$ is zero - as $\alpha$ is outer. The infimum of the left hand side over non-zero projections $e \in R \cap N$ with $e \leq f$ is therefore also zero, as desired.

REMARK. In the situation of Lemma 1 there is a unitary $U_{1} \in R$ such that Ad $U_{1}\left(C_{2}\right) \subset C_{1}^{\prime}$. Then $\gamma\left(\operatorname{Ad} U_{1}\right) \gamma$ is defined as an isomorphism of $R \cap C_{11}^{\prime}$ onto $\quad R \cap C_{21}^{\prime} \quad$ where $\quad C_{11}=C_{1} \vee\left(\left(\operatorname{Ad} U_{1}\right) \gamma\right)^{-1}\left(C_{1}\right) \cong M_{p} \otimes M_{p} \quad$ and $C_{21}=C_{2} \vee \gamma\left(\operatorname{Ad} U_{1}\right)\left(C_{2}\right) \cong M_{q} \otimes M_{q}$. Thus, $\gamma\left(\operatorname{Ad} U_{1}\right) \gamma$ also satisfies the conclusion of Lemma 1. Similarly one can find unitaries $U_{i}$ such that $\gamma\left(\operatorname{Ad} U_{n-1}\right) \gamma\left(\operatorname{Ad} U_{n-2}\right) \cdots\left(\operatorname{Ad} U_{1}\right) \gamma$ is well defined as an isomorphism of $R \cap C_{1 n}^{\prime}$ onto $R \cap C_{2 n}^{\prime}$ where $C_{1 n} \cong M_{p^{n}}$ and $C_{2 n} \cong M_{q^{n}}$. As in [C] and in the introduction, define, for a free ultrafilter $\omega$ on $\mathrm{N}$, the asymptotic centraliser $R_{\omega}$, which is a finite von Neumann algebra. Then the partial endomorphism $\gamma$ of $R$ defines an automorphism $\gamma_{\omega}$ of $R_{\omega}$, and it follows that

$$
\left(\gamma\left(\operatorname{Ad} U_{n-1}\right) \gamma \cdots\left(\operatorname{Ad} U_{1}\right) \gamma\right)_{\omega}=\gamma_{\omega}^{n},
$$

independently of the choice of $U_{1}, U_{2}, \ldots, U_{n-1}$.

Lemma 2. Let $\gamma$ be as in Lemma 1 and let $\gamma_{\omega}, R_{\omega}$ be as above. Then $\gamma_{\omega}^{n}$ is properly outer for any $n \neq 0$.

Proof. Let $N$ be a full matrix subalgebra of $R$ such that $N \supset C_{1}$. As in the proof of $[\mathrm{C}, 1.2 .5]$, for a fixed $\delta>0$ denote by $Q$ the set of couples $(e, V)$ such that

(a) $e$ is a projection in $R \cap N^{\prime}$,

(b) $V$ is a unitary in $R$ with $\|V-1\|_{1} \leq \delta \tau(e)$,

(c) $V \gamma(e) V^{*}$ is a projection in $R \cap N^{\prime}$ and $V \gamma(e) V^{*} e=0$.

We define an order on $Q$ as follows : $(e, V) \leq\left(e^{\prime}, V^{\prime}\right)$ if

(1) $e \leq e^{\prime}$

(2) $\left\|V^{\prime}-V\right\|_{1} \leq \delta \tau\left(e^{\prime}-e\right)$.

One can show that $(Q, \leq)$ is inductive, and, by using Lemma1, that a maximal element $(e, V)$ satisfies $e+V \gamma(e) V^{*}=1$. Since 


$$
\begin{aligned}
\tau\left((e-\gamma(e))^{2}\right) & =\tau(e+\gamma(e)-e \gamma(e)-\gamma(e) e) \\
& =1-2 \tau(e \gamma(e) e) \\
& =1-2 \tau(e(1-V) \gamma(e) e)
\end{aligned}
$$

and

$$
\begin{aligned}
|\tau(e(1-V) \gamma(e) e)| & \leq \tau\left((1-V)(1-V)^{*}\right)^{\frac{1}{2}} \tau(e \gamma(e) e)^{\frac{1}{2}} \\
& \leq \sqrt{2} \tau(|1-V|)^{\frac{1}{2}} \tau(e)^{\frac{1}{2}} \\
& =\tau(|1-V|)^{\frac{1}{2}} \\
& \leq\left(\frac{\delta}{2}\right)^{\frac{1}{2}}
\end{aligned}
$$

we obtain

$$
\|e-\gamma(e)\|_{2} \geq(1-\sqrt{2 \delta})^{\frac{1}{2}}
$$

Hence if $\delta=1 / 8,\|e-\gamma(e)\|_{2} \geq 1 / \sqrt{2}$. For an increasing sequence $\left(N_{n}\right)$ of full matrix subalgebras of $R$ such that $C_{1} \subset N_{1}$ and $\bigcup_{n=1}^{\infty} N_{n}$ is dense in $R$, we choose $e_{n} \in R \cap N_{n}^{\prime}$ as above, and set $e=\left(e_{n}\right) \in R_{\omega}$. Then it follows that $\left\|e-\gamma_{\omega}(e)\right\|_{2} \geq 1 / \sqrt{2}$. Thus we have that $\gamma_{\omega}$ is properly outer as in the proof of [C, 2.1.2]. By the Remark above, this argument also applies to any nonzero power of $\gamma_{\omega}$.

Lemma 3. In the situation of the previous lemma, $\gamma$ has the Rohlin property, i.e., for any $n \in \mathrm{N}, \varepsilon>0$, and finite subset $S$ of $R \cap C_{1}^{\prime}$ there exists a partition $\left(F_{0}, \ldots, F_{n-1}\right)$ of unity by projections in $R \cap C_{1}^{\prime}$ such that

$$
\begin{aligned}
& \left\|\gamma\left(F_{i}\right)-F_{i+1}\right\|_{2}<\varepsilon, \\
& \left\|\left[x, F_{i}\right]\right\|_{2}<\varepsilon
\end{aligned}
$$

for $i=0, \ldots, n-1$ with $F_{n}=F_{0}$ and for $x \in S$, where $\|y\|_{2}=\tau\left(y^{*} y\right)^{\frac{1}{2}}$ for $y \in R$.

Proof. This follows from [C, 1.2.5] in view of [C, 1.1.3].

Lemma 4. Let $B$ be a UHF algebra and let $C_{1}$ and $C_{2}$ be unital finite-dimensional subalgebras of $B$ such that $C_{1} \cong M_{p}$ and $C_{2} \cong M_{q}$ with $p \neq q$. Let $\gamma$ be an isomorphism of $B \cap C_{1}^{\prime}$ onto $B \cap C_{2}^{\prime}$. Then $\gamma$ has the Rohlin property, i.e., for any $n, \varepsilon>0$, and finite subset $S$ of $B \cap C_{1}^{\prime}$ there is a partition $\left(F_{i} ; i=0,1, \ldots,(p q)^{n}-1\right)$ of unity of projections in $B \cap C_{1}^{\prime}$ such that 


$$
\begin{aligned}
& \left\|\gamma\left(F_{i}\right)-F_{i+1}\right\|<\varepsilon, \\
& \left\|\left[x, F_{i}\right]\right\|<\varepsilon
\end{aligned}
$$

for $i=0,1, \ldots,(p q)^{n}-1$ with $F_{(p q)^{n}}=F_{0}$ and for $x \in S$.

Proof. Let $N$ be a full matrix subalgebra of $B$ with $N \supset C_{1}$. For any $n$ and $\varepsilon>0$ we have to construct a partition $\left(F_{i} ; i=0,1, \ldots,(p q)^{n}-1\right)$ of unity in $B \cap N^{\prime}$ such that

$$
\left\|\gamma\left(F_{i}\right)-F_{i+1}\right\|<\varepsilon
$$

for $i=0,1, \ldots,(p q)^{n}-1$. By having Lemma 3 at hand this can be done exactly as in [K2].

Lemma 5. In the situation of the previous lemma, let $\left(U_{n}\right)$ be a central sequence of unitaries in $B$. Then there exists a central sequence $\left(V_{n}\right)$ of unitaries in $B \cap C_{1}^{\prime}$ such that

$$
\left\|\gamma\left(V_{n}^{*}\right) V_{n}-U_{n}\right\| \rightarrow 0
$$

Proof. Set $A^{\infty}=l^{\infty}(\mathrm{N}, A) / c_{0}(\mathrm{~N}, A)$ and imbed $A$ into $A^{\infty}$ as the constant sequences. Set $A_{\infty}=A^{\infty} \cap A^{\prime}$. Then $\gamma$ defines an automorphism $\gamma_{\infty}$ of $A_{\infty}$ and by Lemma $4, \gamma_{\infty}$ has the Rohlin property. Hence by [HO] we have a unitary $v \in A_{\infty}$ for $u=\left(U_{n}\right) \in A_{\infty}$ such that

$$
\gamma_{\infty}\left(v^{*}\right) v=u \text {. }
$$

We may represent $v$ as a sequence $\left(V_{n}\right)$ of unitaries in $B \cap C_{1}^{\prime}$. This completes the proof.

Lemma 6. In the situation of Lemma 4, consider in addition the UHF algebra $B_{0}=M_{(p q)}$ and let $\sigma$ be an automorphism of $B_{0}$. Then $\gamma$ and $\gamma \otimes \sigma$ are outer conjugate; that is, there is an isomorphism $\varphi$ of $B \otimes B_{0}$ onto $B$ such that $\varphi\left(C_{1} \otimes 1\right)=C_{1}$ and

$$
(\operatorname{Ad} U) \gamma=\varphi(\gamma \otimes \sigma) \varphi^{-1} \text { on } B \cap C_{1}^{\prime}
$$

for some unitary $U \in B$.

Proof. First note that $B \cong B \otimes B_{0}$. Therefore, we may choose a central sequence $\left(e_{i j}^{n} ; i, j=1, \ldots, p q\right)$ of $p q \times p q$ matrix units in $B \cap C_{1}^{\prime}$. Choose an element $\lambda$ of T of infinite order. Since $\gamma$ takes $B \cap C_{1}^{\prime}$ onto $B \cap C_{2}^{\prime}$, there exists a central sequence $\left(v_{n}\right)$ of unitaries in $B$ such that

$$
\gamma\left(e_{i j}^{n}\right)=\lambda^{i-j} v_{n} e_{i j}^{n} v_{n}^{*} .
$$

By Lemma 5, there exists a central sequence $\left(w_{n}\right)$ of unitaries in $B \cap C_{1}^{\prime}$ such 
that $\left\|\gamma\left(w_{n}^{*}\right) w_{n}-v_{n}\right\| \rightarrow 0$. Set $f_{i j}^{n}=w_{n} e_{i j}^{n} w_{n}^{*}$. Then $\left(f_{i j}^{n}: i, j=1, \ldots, p q\right)$ is a central sequence of systems of $p q \times p q$ matrix units in $B \cap C_{1}^{\prime}$, and

$$
\gamma\left(f_{i j}^{n}\right)-\lambda^{i-j} f_{i j}^{n} \rightarrow 0 .
$$

We may choose a subsequence of $\left(f_{i j}^{n}\right)$ and modify it slightly so that the resulting sequence $\left(f_{i j}^{n}\right)$ consists of mutually commuting systems of matrix units and there is a unitary $U \in B$ with

$$
(\operatorname{Ad} U) \gamma\left(f_{i j}^{n}\right)=\lambda^{i-j} f_{i j}^{n} .
$$

This implies that $B$ has a factorization $B_{1} \otimes B_{0}$ with respect to which (Ad $U$ ) $\gamma$ factorizes as $\gamma_{1} \otimes \sigma_{0}$, where $\gamma_{1}$ is a partially defined endomorphism of $B_{1}$ of the same type as $\gamma$ and $\sigma_{0}$ is the automorphism of $B_{0}$ consisting of the infinite tensor product of copies of

$$
\operatorname{Ad}\left(\begin{array}{cccc}
1 & & & 0 \\
0 & \lambda & & 0 \\
& & \ddots & \\
0 & & & \lambda^{p q-1}
\end{array}\right) .
$$

Since $\sigma_{0}$ has the Rohlin property ([BEK2], [K1]), for any automorphism $\sigma$ of $B_{0}$ there is (by [K2]) a unitary $V$ in $B_{0} \otimes B_{0}$ such that

$$
\sigma_{0} \cong \operatorname{Ad} V\left(\sigma_{0} \otimes \sigma\right),
$$

where $\cong$ denotes conjugacy. This implies that

$$
(\operatorname{Ad} U) \gamma \cong \operatorname{Ad}(1 \otimes V)\left(\gamma_{1} \otimes \sigma_{0} \otimes \sigma\right) \cong \operatorname{Ad}(\widetilde{V}(U \otimes 1)) \gamma \otimes \sigma
$$

where $\widetilde{V}$ denotes the image of $1 \otimes V$ under the second isomorphism. This completes the proof.

THEOREM 7. Let $A$ be a stable UHF algebra and let $\alpha, \beta$ be automorphisms of $A$. If $s(\alpha)=s(\beta) \neq 1$, then $\alpha$ and $\beta$ are outer conjugate.

Proof. Let $A \cong B \otimes \mathcal{K}$ with $B$ a unital UHF algebra and write $s(\alpha)=p / q$ with $p$ and $q$ relatively prime positive integers. As shown above we may assume that $\alpha$ has the decomposition

$$
A=B \otimes \mathcal{K}=B \cap C_{1}^{\prime} \otimes\left(C_{1} \otimes \mathcal{K}\right) \gamma \otimes \theta \longrightarrow B \cap C_{2}^{\prime} \otimes\left(C_{2} \otimes \mathcal{K}\right)=A,
$$

where $\gamma$ is an isomorphism of $B \cap C_{1}^{\prime}$ onto $B \cap C_{2}^{\prime}, C_{1} \cong M_{p}, C_{2} \cong M_{q}$, and $\theta$ is an isomorphism of $C_{1} \otimes \mathcal{K}$ onto $C_{2} \otimes \mathcal{K}$. We may identify $\mathcal{K}$ with $\mathcal{K} \otimes \mathcal{K}$. Since $\theta$ is outer conjugate to $\theta \otimes \mathrm{id}_{\mathcal{K}}$, this implies that $\alpha$ is outer conjugate to $\alpha \otimes \operatorname{id}_{\mathcal{K}}$.

By Lemma 6 applied to $\gamma, \alpha$ is outer conjugate to $\alpha \otimes \sigma$ for any automorphism $\sigma$ of $B_{0}=M_{(p q)}$. 
Let $\nu$ be an automorphism of $B_{0} \otimes \mathcal{K}$ such that $s(\nu)=s(\alpha)$. Then $s\left(\alpha \otimes \nu^{-1}\right)=1$, and so $\alpha \otimes \nu^{-1}$ is outer conjugate to $\sigma \otimes \operatorname{id}_{\mathcal{K}}$ on $B \otimes \mathcal{K}$ for some automorphism $\sigma$ of $B$. By Lemma 4 applied to $\gamma$, it follows that $\sigma$ has the Rohlin property and so $\alpha \otimes \nu^{-1}$ is outer conjugate to $\sigma_{0} \otimes \mathrm{id}_{B} \otimes \mathrm{id}_{\mathcal{K}}$ on $B_{0} \otimes B \otimes \mathcal{K}$ with $\sigma_{0}$ an automorphism of $B_{0}$ with the Rohlin property. Similarly, $\nu \otimes \nu^{-1}$ is outer conjugate to $\sigma_{0} \otimes \mathrm{id}_{\mathcal{K}}$. Thus we have that

$$
\begin{aligned}
\alpha & \sim \alpha \otimes \operatorname{id}_{\mathcal{K}} \sim \alpha \otimes \sigma_{0} \otimes \operatorname{id}_{\mathcal{K}} \sim \alpha \otimes \nu \otimes \nu^{-1} \otimes \operatorname{id}_{\mathcal{K}} \\
& \sim \nu \otimes \sigma_{0} \otimes \operatorname{id}_{B} \otimes \operatorname{id}_{\mathcal{K}} \sim \nu \otimes \sigma_{0} \otimes \operatorname{id}_{B} \sim \nu \otimes \operatorname{id}_{B},
\end{aligned}
$$

where outer conjugacy is denoted by $\sim$. Since the right hand side does not depend on $\alpha$, we have the assertion.

REMARK. Let $A$ be a stable UHF algebra and let $\alpha$ be an automorphism of $A$ with $s(\alpha) \neq 1$. Then by Theorem 7 the isomorphism class of the crossed product $A \rtimes_{\alpha} \mathrm{Z}$ depends only on $s(\alpha)$. Since $A \rtimes_{\alpha} \mathrm{Z}$ is purely infinite [R2], this fact also follows from [R2], [Kir] and [Phi], by using the Pimsner-Voiculescu exact sequence. When $A=M_{(p q)^{\infty}} \otimes \mathcal{K}$ and $s(\alpha)=p / q$, where $p, q$ are relatively prime, calculation yields that

$$
K_{0}\left(A \rtimes_{\alpha} \mathbf{Z}\right)=\mathbf{Z} /(p-q) \mathbf{Z}, \quad K_{1}\left(A \rtimes_{\alpha} \mathbf{Z}\right)=0 .
$$

Hence by [Kir] and [Phi], $A \rtimes_{\alpha} \mathrm{Z}$ is isomorphic to the stable Cuntz algebra $O_{|p-q|+1} \otimes \mathcal{K}$.

COROllary 8. Let $\alpha$ and $\beta$ be unital endomorphisms of a UHF algebra $B$ such that $\operatorname{Im} \alpha=B \cap B_{\alpha}^{\prime}$ and $\operatorname{Im} \beta=B \cap B_{\beta}^{\prime}$ where $B_{\alpha}$ and $B_{\beta}$ are unital subalgebras of $B$ with $B_{\alpha} \cong M_{p} \cong B_{\beta}$ with $p \geq 2$. Then $\alpha$ and $\beta$ are outer conjugate.

Proof. Denote by $\sigma$ the unilateral shift on $M_{p^{\infty}}$ and by $B_{\sigma}$ the first copy of $M_{p}$ in $M_{p^{\infty}}$. Then $\operatorname{Im} \sigma=M_{p^{\infty}} \cap B_{\sigma}^{\prime}$ and $\sigma$ is an endomorphism of $M_{p^{\infty}}$ of the type considered in the corollary.

We define an automorphism $\left(\alpha^{-1}, \sigma\right)$ of $B \otimes M_{p^{\infty}}$ by

$$
B \otimes M_{p^{\infty}}=\alpha(B) \otimes B_{\alpha} \otimes M_{p^{\infty}} \stackrel{\alpha^{-1} \otimes \theta_{\alpha \sigma} \otimes \sigma}{\longrightarrow} B \otimes B_{\sigma} \otimes \sigma\left(M_{p^{\infty}}\right)=B \otimes M_{p^{\infty}}
$$

where $\theta_{\alpha \sigma}$ is an isomorphism of $B_{\alpha}$ onto $B_{\sigma}$. Thus, $\left(\alpha^{-1}, \sigma\right)$ is defined uniquely up to inner automorphisms. Similarly we define an automorphism $\left(\alpha, \sigma^{-1}\right)$ of $B \otimes M_{p^{\infty}}$, which is the inverse of $\left(\alpha^{-1}, \sigma\right)$ up to inner automorphisms, and also an automorphism $\left(\sigma^{-1}, \sigma\right)$ of $M_{p^{\infty}} \otimes M_{p^{\infty}}$.

Since, by Lemma $4, \sigma$ has the Rohlin property, $\left(\sigma^{-1}, \sigma\right)$ also has the Rohlin property and so by [K2] is outer conjugate to any other automorphism of $M_{p^{\infty}}$ with the Rohlin property. Hence by Lemma 6 ,

$$
\alpha \sim \alpha \otimes\left(\sigma^{-1}, \sigma\right) \sim\left(\alpha, \sigma^{-1}\right) \otimes \sigma \sim \sigma \otimes \operatorname{id}_{B},
$$


where the second outer conjugacy consists of exchanging $B_{\sigma}$ in the last factor and $B_{\alpha}$ in the first factor of the factorisation $B \otimes M_{p^{\infty}} \otimes M_{p^{\infty}}$, which is an inner automorphism. Since the right hand side does not depend on $\alpha$, this completes the proof.

COROLlaRY 9. If $\alpha$ and $\beta$ are unital endomorphisms of the hyperfinite type $\mathrm{II}_{1}$ factor $R$ such that $\operatorname{Im} \alpha=R \cap R_{\alpha}^{\prime}$ and $\operatorname{Im} \beta=R \cap R_{\beta}^{\prime}$ for some unital finitedimensional subfactors $R_{\alpha}$ and $R_{\beta}$ of $R$, then $\alpha$ and $\beta$ are outer conjugate if and only if $R_{\alpha} \cong R_{\beta}$.

Proof. This is proved in exactly the same way as the previous corollary.

REMARK. If $M$ is a type $I_{\infty}$ factor and $\alpha$ is a unital normal endomorphism of $M$, then $M_{\alpha}=M \cap \alpha(M)^{\prime}$ is of type $\mathrm{I}_{n}$ for some $n$ and $\alpha(M)=M \cap M_{\alpha}^{\prime}$. The outer conjugacy class of $\alpha$ is uniquely determined by $n$.

REMARK. Let $M$ be a properly infinite factor and let $\alpha$ be a unital normal endomorphism of $M$ such that $\alpha(M)=M \cap M_{\alpha}^{\prime}$ for some type I subfactor $M_{\alpha}$ of $M$. Suppose that $M_{\alpha}$ is of type $\mathrm{I}_{n_{\alpha}}$ (with $n_{\alpha}$ countable). Then there exist $n_{\alpha}$ isometries $\left(s_{i}\right)$ in $M$ such that $\left(s_{i} s_{j}^{*}\right)$ is a system of matrix units for $M_{\alpha}$, and $\alpha$ is given by

$$
\alpha(x)=\sum s_{i} \gamma(x) s_{i}^{*}
$$

for some automorphism $\gamma$ of $M$. For a different choice of $\left(s_{i}\right), \gamma$ differs only by an inner automorphism determined by a unitary in $M_{\alpha}$. Denote the class containing $\gamma$ in Aut $M /$ Int $M$ by $\hat{\alpha}$. Then we can assert: Two such endomorphisms $\alpha, \beta$ of $M$ are outer conjugate if and only if $n_{\alpha}=n_{\beta}$ and $\hat{\alpha}$ is conjugate to $\hat{\beta}$. (If $M$ is of type III or type $\mathrm{II}_{\infty}$, it is known [C] that there is an abundance of outer conjugacy classes in Aut $M$; so the situation is different from the one in Corollary 9.) For example if $\alpha$ and $\gamma$ are as above and $\sigma$ is another automorphism of $M$, then

$$
\sum s_{i} \sigma \gamma \sigma^{-1}(x) s_{i}^{*}=(\operatorname{Ad} V) \sigma \alpha \sigma^{-1}(x)
$$

where $V$ is the unitary defined by

$$
V=\sum s_{i} \sigma\left(s_{i}\right)^{*} .
$$

The other computations are as easy as this.

REMARK. With $\sigma$ the unilateral shift endomorphism of the UHF algebra $M_{2^{\infty}}$, define an endomorphism $\alpha$ of $M_{2^{\infty}}$ by

$$
\alpha(x)=e_{1} \sigma(x)+e_{2} \sigma^{2}(x)
$$


where $e_{1}, e_{2}$ is a partition of unity by projections in the first copy of $M_{2}$ in $M_{2^{\infty}}$. Note that the relative commutant of $\operatorname{Im} \alpha$ is isomorphic to $\mathrm{C} \oplus M_{2}$, and that $\alpha$ does not have the Rohlin property. For if it did, then there would be a unitary $u \in M_{2 \infty}$ such that $u$ commutes with $M_{2}$ and $\alpha(u) \approx-u$, which implies that $\sigma(u) \approx-u$ and $\sigma^{2}(u) \approx-u$, a contradiction. When $\gamma$ is an automorphism of $M_{2^{\infty}}$ such that $\sigma \gamma=\gamma \sigma$ and the action of $\mathbf{N} \times \mathbf{Z}$ defined by $(m, n) \rightarrow \sigma^{m} \gamma^{n}$ satisfies the Rohlin property (cf. Remark 4 of [KK]), define an endomorphism $\beta$ of $M_{2 \infty}$ by

$$
\beta(x)=e_{1} \sigma(x)+e_{2} \gamma \sigma^{2}(x) .
$$

Then it follows that $M_{2^{\infty}} \cap(\operatorname{Im} \beta)^{\prime}=M_{2^{\infty}} \cap(\operatorname{Im} \alpha)^{\prime}$ and that $\beta$ has the Rohlin property. Compare with Lemma 4 and Corollary 8.

\section{REFERENCES}

[BEK1] O. Bratteli, D. E. Evans and A. Kishimoto, Almost shift invariant projections in infinite tensor products. Quantum and Non-Commutative Analysis (eds. H. Araki et al.), 427-434, 1994.

[BEK2] O. Bratteli, D. E. Evans and A. Kishimoto (1995), The Rohlin property for quasi-free automorphisms of the Fermion algebra. Proc. London Math. Soc. 71 (1995), 675694.

[BKRS] O. Bratteli, A. Kishimoto, M. Rørdam and E. Størmer (1993), The crossed product of a UHF algebra by a shift. Ergodic Theory Dynamical Systems 13, (1993), 615-626.

[C] A. Connes, Outer conjugacy classes of automorphisms of factors, Ann. Sci. École Norm. Sup. 8 (1975), 383-420.

[CE] A. Connes and D. E. Evans, Embeddings of U(1)-current algebras in non-commutative algebras of classical statistical mechanics. Comm. Math. Phys. 121 (1989), 507-525.

[CT] A. Connes and M. Takesaki, The flow of weights on factors of type III. Tôhoku Math. J. 29 (1977), 473-575.

[HJ1] R. H. Herman and V. F. R. Jones, Period two automorphisms of UHF algebras. J. Funct. Anal. 45 (1982), 169-176.

[HJ2] R. H. Herman and V. F. R. Jones, Models of finite group actions. Math. Scand. 52 (1983), 312-320.

[HO] R. H. Herman and A. Ocneanu, Stability for integer actions on UHF $C^{*}$-algebras, J. Funct. Anal. 59 (1984), 132-144.

[J] V. F. R. Jones, Actions of finite groups on the hyperfinite type $\mathrm{II}_{1}$ factor. Mem. Amer. Math. Soc. 237 (1980).

[KST] Y. Kawahigashi, C. E. Sutherland and M. Takesaki, The structure of the automorphism group of an injective factor and the cocycle conjugacy of discrete abelian group actions. Acta Math. 169 (1992), 105-130.

[Kir] E. Kirchberg (in preparation), The classification of purely infinite $C^{*}$-algebras using Kasparov's theory.

[K1] A. Kishimoto, The Rohlin property for shifts on UHF algebras and automorphisms of Cuntz algebras, J. Funct. Anal. 140 (1995), 100-123.

[K2] A. Kishimoto, The Rohlin property for automorphisms of UHF algebras. J. Reine Angew. Math. 465 (1995), 183-196.

[KK] A. Kishimoto and A. Kumjian, Crossed products of Cuntz algebras by quasi-free automorphisms. Operator algebras and their applications, Fields Institute Commun. 13 (1997), 173-192. 
[O] A. Ocneanu, Actions of discrete amenable groups on factors. Lecture Notes in Math. 1138, 1985.

[Phi] N. C. Phillips, A classification theorem for nuclear purely infinite simple $C^{*}$-algebras (1995).

[R1] M. Rørdam, Classification of inductive limits of Cuntz algebras. J. Reine Angew. Math. 440 (1993), 175-200.

[R2] M. Rørdam, Classification of certain infinite simple $C^{*}$-algebras. J. Funct. Anal. 131 (1995), 415-458.

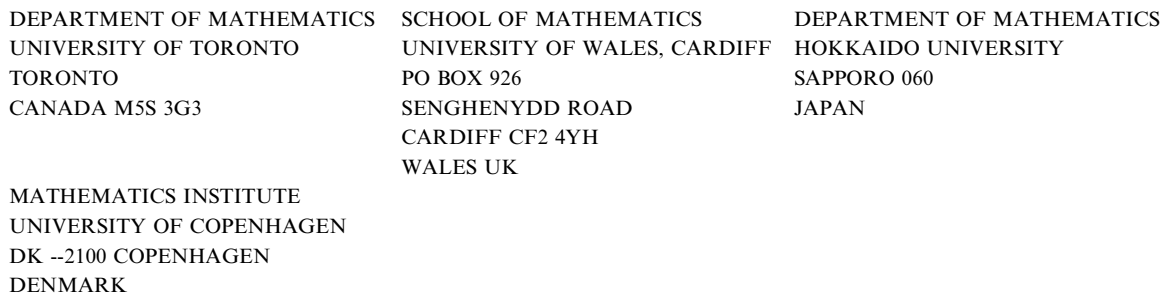

